\title{
Development of Dedicated Brain PET Imaging Devices: Recent Advances and Future Perspectives
}

\author{
Ciprian Catana
}

Athinoula A. Martinos Center for Biomedical Imaging, Department of Radiology, Massachusetts General Hospital and Harvard Medical School, Charlestown, Massachusetts

\begin{abstract}
Whole-body PET scanners are not optimized for imaging small structures in the human brain. Several PET devices specifically designed for this task have been proposed either for stand-alone operation or as MR-compatible inserts. The main distinctive features of some of the most recent concepts and their performance characteristics, with a focus on spatial resolution and sensitivity, are reviewed. The tradeoffs between the various performance characteristics, desired capabilities, and cost that need to be considered when designing a dedicated brain scanner are presented. Finally, the aspirational goals for future-generation scanners, some of the factors that have contributed to the current status, and how recent advances may affect future developments in dedicated brain PET instrumentation are briefly discussed.
\end{abstract}

Key Words: PET; neuroimaging; high spatial resolution; multimodal imaging

J Nucl Med 2019; 60:1044-1052

DOI: 10.2967/jnumed.118.217901

\section{$\mathbf{P}$}

ET provides unique information about the human brain that has proved useful in a variety of studies ranging from basic neuroscience to clinical applications. In parallel with the development of dozens of radiotracers and the implementation of methods for extracting ever-more-accurate estimates of parameters related to many biologic processes (1), PET instrumentation has continuously evolved over the last 6 decades (2). In addition to general-purpose whole-body devices, several scanners designed specifically for studying the brain have also been developed. This review will focus mainly on those concepts proposed over the last decade and a half and will not cover earlier-generation devices that were equally innovative at their time (e.g., Positome II (3), NeuroECAT (4), Head PENN-PET (5), Moses et al. (6), Hamamatsu SHR12000 (7), and G-PET (8)). Similarly, the scanners developed specifically for small-animal imaging will not be discussed here, although they share many similarities with the dedicated human brain scanners in terms of the design constraints and performance requirements $(9)$.

The development of dedicated brain PET hardware was initially motivated by the need for improved performance compared with

Received Feb. 3, 2019; revision accepted Apr. 16, 2019.

For correspondence or reprints contact: Ciprian Catana, Athinoula A. Martinos Center for Biomedical Imaging, Building 149, 13th St., Room 2.301, Charlestown, MA 02129.

E-mail: ccatana@mgh.harvard.edu

Published online Apr. 26, 2019.

COPYRIGHT (C 2019 by the Society of Nuclear Medicine and Molecular Imaging. whole-body devices, specifically better spatial resolution or higher sensitivity to enable the imaging of small brain structures. The spatial resolution in PET depends on several factors ranging from physics limitations related to the positron emission and annihilation (i.e., positron range and noncollinearity) to those dependent on the detection system (e.g., crystal size, photon detector, and scanner diameter) or the image reconstruction algorithm (10). The contributions of most of these factors could be reduced in a dedicated brain scanner. For example, decreasing the scanner diameter to better match the size of the human head minimizes the contribution of the noncollinearity effect. Even the in-plane positron range error could be reduced by placing the PET scanner in a high-magnetic field, which would arguably be easier to accomplish for a more compact design. The PET scanner sensitivity depends mainly on the solid-angle coverage and photon detection efficiency. Reducing the diameter of the scanner and increasing the area of the detector improve the former, whereas using thicker scintillation crystals with high linear attenuation coefficients improves the latter.

In addition to achieving better performance, other groups were motived by the desire to improve the portability, mobility, or wearability of the device. Furthermore, given the smaller overall size, the integration with MRI scanners is also easier, and this and other nontechnical reasons have led to the development of MRcompatible PET inserts. Finally, reducing the cost of the scanner compared with whole-body devices was another motivation, although this might not necessarily be the case when the performance is also improved. In fact, developing dedicated brain PET scanners is all about making trade-offs between the various performance characteristics, desired capabilities, and cost. The most obvious trade-off is that between sensitivity and spatial resolution; using longer crystals to improve the former degrades the latter toward the edge of the field of view (FOV) because of the parallax error unless the detectors have depth-of-interaction (DOI) encoding capabilities (i.e., able to estimate the depth in the detector at which the annihilation photons are absorbed).

The dedicated brain PET devices discussed in the following sections have been developed either for stand-alone operation or as MR-compatible inserts. In each of these two categories, the representative scanner that was used for the largest number of human studies is discussed first. Furthermore, two subcategories based on the scanner geometry are defined. Those devices with a constant number of detectors along the axial extent (e.g., the typical cylindrical geometry scanners) belong to the conventional subcategory, whereas those with other geometries belong to the unconventional subcategory. In each case, the main design and performance characteristics are summarized, with a focus on spatial resolution, sensitivity, and distinctive features. 


\section{STAND-ALONE PET SCANNERS}

\section{Conventional Scanner Geometry Devices}

Perhaps the best-known dedicated brain PET scanner and one of the few that has been commercialized is the High-Resolution Research Tomograph (HRRT) built by CTI PET Systems, Inc. (Fig. 1A). The main design goal for the HRRT was a spatial resolution of at least $2.5 \mathrm{~mm}$ across the human brain. For this purpose, a compact scanner geometry was chosen (the electronic axial and transaxial FOVs were 25.2 and $31.2 \mathrm{~cm}$, respectively), and detector blocks with DOI capability were used to minimize the spatial resolution variation across the imaging volume. Specifically, 2 layers of 64 lutetium oxyorthosilicate crystals $\left(2.1 \times 2.1 \times 7.5 \mathrm{~mm}^{3}\right)$ with different light decay times were read out using photomultiplier tubes with a quadrant-sharing detector block design (11). Pulse shape discrimination allowed the assignment of each event to one of the two layers. Eight panel detector heads were arranged in an octagon, with opposing heads positioned $46.9 \mathrm{~cm}$ apart (Fig. 2A). The spatial resolution in the transaxial direction ranged from $2.4 \mathrm{~mm}$ at the center of the FOV to $2.8 \mathrm{~mm}$ at $10 \mathrm{~cm}$ off-center, and the sensitivity was $4.3 \%$ at the center of the FOV (12). The 17 HRRTs installed around the world have been used for numerous studies and allowed neuroscientists and clinical researchers to begin to explore the benefits of higher-spatial-resolution PET imaging (13-18).

To further increase the sensitivity without degrading the spatial resolution, investigators from Japan built the jPET-D4 prototype scanner (Fig. 1B) using a 4-layer DOI detector $(19,20)$. The gantry of the jPET-D4 prototype with an inner diameter of $39 \mathrm{~cm}$ and an axial extent of $26 \mathrm{~cm}$ was made of 5 rings of 24 detector blocks, each consisting of 4 layers of $16 \times 16$ arrays of gadolinium orthosilicate crystals $\left(2.9 \times 2.9 \times 7.5 \mathrm{~mm}^{3}\right)$ (Fig. 2B). The DOI information was obtained by varying both the cerium dopant (different concentrations in the upper and lower 2 layers) and the reflector material placement. The scintillator arrays were read out by 256-channel flat-panel photomultiplier tubes (Hamamatsu Photonics K.K.). The jPET-D4 prototype had a relatively uniform spatial resolution of $3 \mathrm{~mm}$ and an approximately $11 \%$ sensitivity at the center of the FOV.

Increasing the mobility while maintaining high sensitivity and good spatial resolution were the main design goals for the NeuroPET/CT scanner (Fig. 1C) developed by Photo Diagnostic Systems, Inc., another device that was commercialized (21). The first goal was achieved by using electronics that required only standard power and self-propelling the scanner on battery-powered wheels. To improve the sensitivity, the detector ring diameter and axial FOV were 35.7 and $22 \mathrm{~cm}$, respectively. Two layers of

\section{NOTEWORTHY}

- Several concepts have been proposed for developing standalone and MR-compatible dedicated brain PET scanners.

- These scanners have increased performance (e.g., better spatial resolution, higher sensitivity) compared with wholebody devices.

- Recently proposed concepts offer more versatility in performing novel studies.

- Sustained effort is needed for moving from promising concepts to useful products.

- Integration of several imaging modalities will be needed to further our understanding of the human brain and address the many unmet needs in neuropsychiatry.

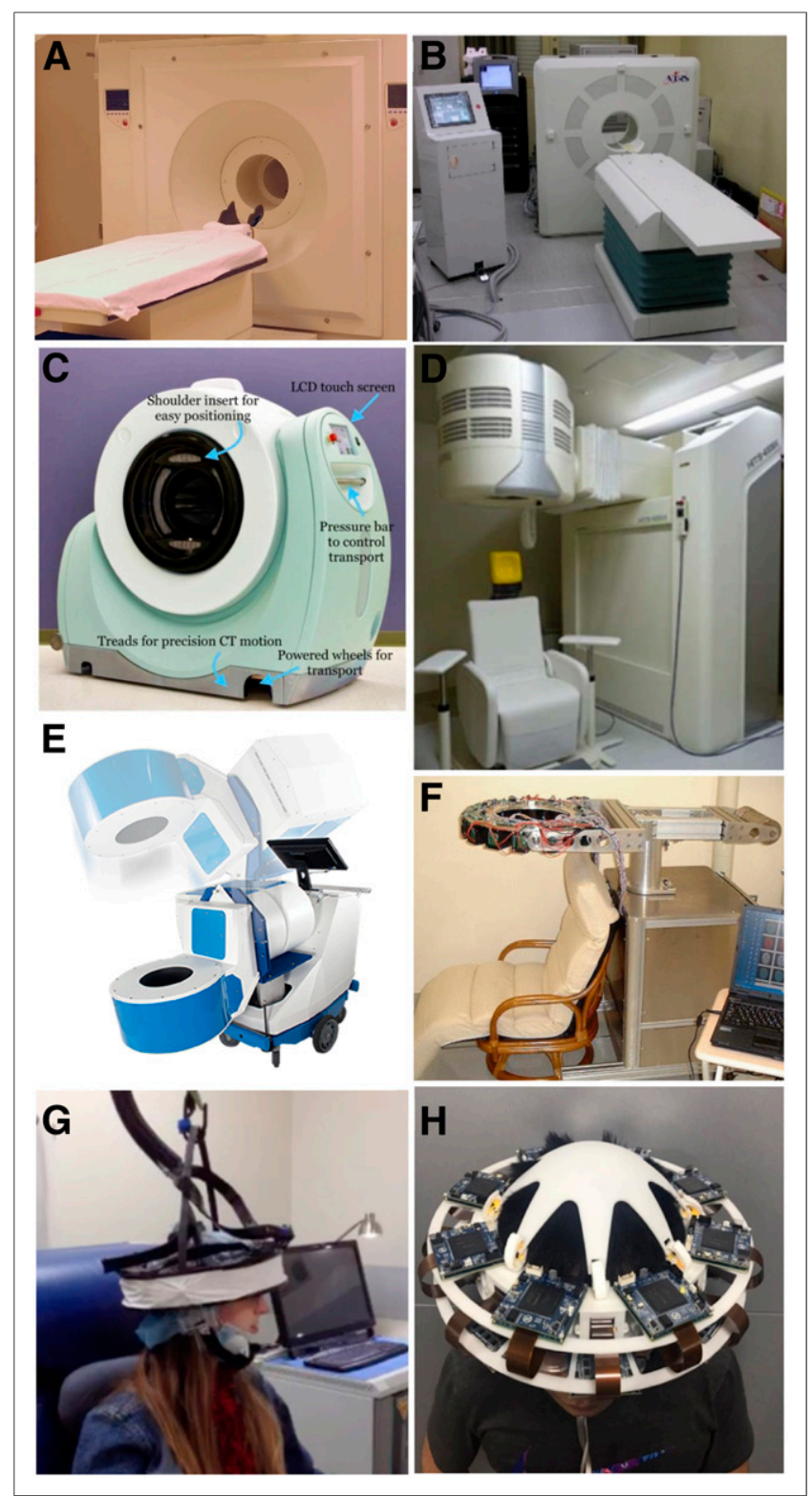

FIGURE 1. Examples of dedicated PET scanners with conventional geometries: HRRT (https://commons.wikimedia.org/wiki/File:HRRT_ PET.JPG) (A), jPET-D4 (20) (B), NeuroPET/CT (22) (C), Hamamatsu (23) (D), BBX (http://prescient-imaging.com/products/bbX) (E), PET-Hat (24) (F), Helmet-PET (27) (G), and Mind-Tracker (courtesy of Qiyu Peng, Lawrence Berkeley National Laboratory) $(\mathrm{H})$.

cerium-doped lutetium yttrium orthosilicate (LYSO) crystals (2.3 $\times 2.3 \times 10 \mathrm{~mm}^{3}$ ) offset by one crystal were used to obtain DOI information (Fig. 2C). Silicon photomultipliers (SiPMs) were used as photodetectors. The spatial resolution of the NeuroPET/CT scanner ranged from about $3 \mathrm{~mm}$ at the center of the FOV to 5.2 at $10 \mathrm{~cm}$ from the center. The sensitivity at the center of the FOV was $0.75 \%$ (increasing to $1.16 \%$ for wider energy and timing windows). The NeuroPET/CT scanner is the only device discussed that also had a CT component (3,264 detector channels; 100-140 $\mathrm{kVp}$ and 2-7 mA x-ray source) (22).

Building on their extensive experience developing PET technology, researchers from Hamamatsu Photonics K.K. also developed a high-spatial-resolution dedicated brain scanner with 4-layer DOI 


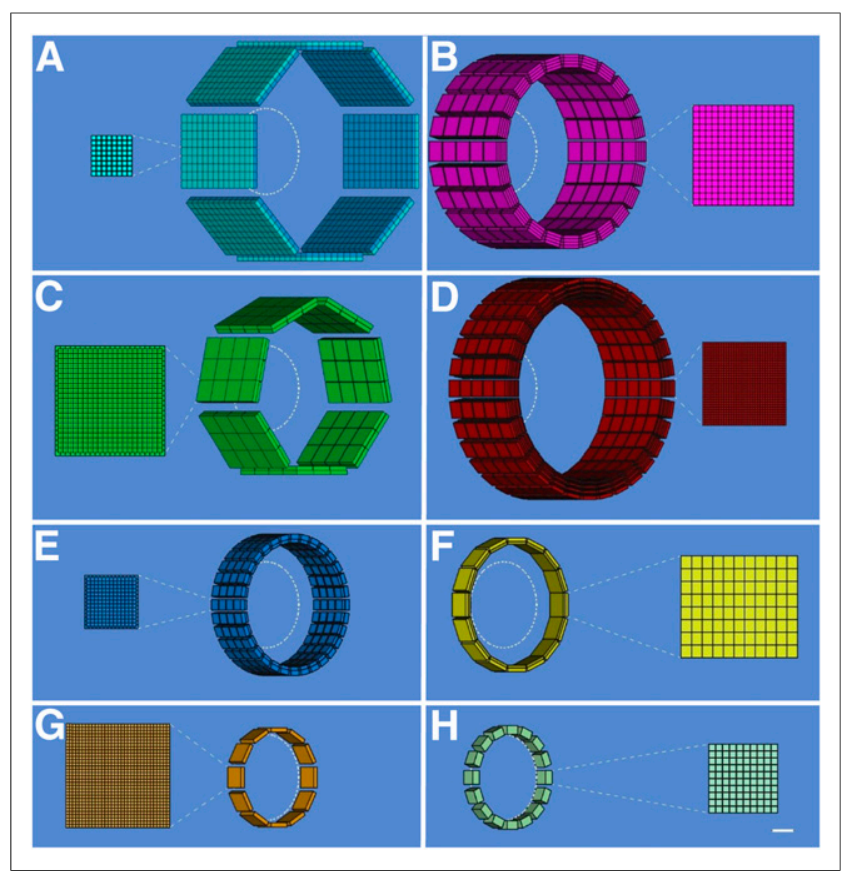

FIGURE 2. Schematic drawings (angled view) showing configuration of detector modules and cross-sectional detailed views (scale, 5:1) of scintillator arrays for dedicated PET scanners with conventional geometries: HRRT (A), jPET-D4 (B), NeuroPET/CT (C), Hamamatsu (D), BBX $(\mathrm{E})$, PET-Hat (F), Helmet-PET (G), and Mind-Tracker $(\mathrm{H})$. A 20-cm-diameter circle is shown inside each scanner. Scale bar represents $1 \mathrm{~cm}$ in detailed views.

detectors (Fig. 1D) (23). Each detector module consisted of 4 detector layer boards with arrays of LYSO crystals $(32 \times 32$ with $1.2-\mathrm{mm}$ pitch) and multipixel photon counters (MPPCs), front-end and signalprocessing circuits, a data interface board, and power supplies. The crystal length varied from 3 to $8 \mathrm{~mm}$ in each layer, for a total of $20 \mathrm{~mm}$. Thirty-two detector modules were positioned in a cylindrical gantry head with transaxial and axial FOVs of 33 and $20.16 \mathrm{~cm}$, respectively (Fig. 2D). The gantry head could move up, down, forward, and backward and could tilt, allowing great flexibility in patient positioning (e.g., scanning with the subject seated). The reconstructed spatial resolution was about $2 \mathrm{~mm}$ throughout the whole FOV, and the absolute sensitivity was about $2 \%$. Phantom, preclinical, and clinical studies were performed, demonstrating excellent performance.

A scanner that combines the distinctive features of the previous two devices was developed by Prescient Imaging, LLC (Hawthorne, California). In addition to portability, the manufacturer's goal was to build an adjustable scanner that allows brain imaging with the patient seated, breast imaging with the patient prone, and extremities imaging with the patient in other positions. Their BBX scanner (Fig. 1E) was designed to fit through standard doors and elevators, and weighed about $225 \mathrm{~kg}$. The detectors consisted of double-layer staggered lutetium fine silicate crystals $(13 \times 13$ and $14 \times 14$ arrays, $1.76-\mathrm{mm}$ pitch) coupled to MPPCs and readout electronics. One hundred twenty-eight blocks positioned in a circular shape make up the gantry, with 288-mm and 100-mm transaxial and axial FOVs, respectively (Fig. 2E). The preliminary evaluation showed good performance, with a spatial resolution of about $2.6 \mathrm{~mm}$ and $2.59 \%$ sensitivity at the center of the FOV.

To give the subject even more freedom to move around during the examination, Yamamoto et al. proposed a wearable design, called PET-Hat (Fig. 1F), that allowed partial head movement using a double counter-balanced mechanical support system (24). A stand was used to support two arms that held the PET detector ring and the counterbalancing weight. This system permitted almost free range of movement because the arms allowed the up-and-down motion of the detector ring and the stand could rotate around the base. The DOI PET detector block consisted of 2 layers of gadolinium orthosilicate crystals $\left(4.9 \times 5.9 \times 7\right.$ and $\left.4.9 \times 5.9 \times 8 \mathrm{~mm}^{3}\right)$ doped with different concentrations of cerium, a tapered light guide, and a photomultiplier tube. As another trade-off, the total length of the scintillator material was chosen to minimize the weight, increase safety, and decrease inertia. Sixteen detector blocks were arranged in a 280-mm-diameter ring (Fig. 2F). The whole PET-Hat scanner could be mounted on a straw hat to be worn by the subject. The spatial resolution was below $4.2 \mathrm{~mm}$ throughout the FOV, and the sensitivity for a point source was $0.72 \%$ at the center of the FOV. Although small structures could be observed in the images obtained with a Hoffman brain phantom, the image quality was affected by the low sensitivity and the low noise-equivalent counting rate because of high scatter and random ratios.

To allow the subjects to be scanned standing or moving, the wearable Helmet-PET and ambulatory microdose PET concepts (Fig. 1G) were proposed $(25,26)$. The original design consisted of 25 detector modules each made up of 2 LYSO arrays $(1 \times 1 \times 10$ and $1.5 \times 1.5 \times 10 \mathrm{~mm}^{3}$ ) coupled to $5 \times 5$ arrays of MPPCs. Subsequently, these concepts of wearable PET imagers that could eventually be used for monitoring motor tasks, artistic or creative tasks, and complex social interactions have continued to evolve (27). The next prototype consisted of a ring of 12 detector modules supported by a flexible mechanical mount. Each module had a $32 \times$ 32 array of $1.5 \times 1.5 \times 10 \mathrm{~mm}^{3}$ LYSO crystals coupled to MPPCs through a light guide (Fig. 2G). This prototype was used for scanning phantoms and 4 human subjects, studies that allowed the investigators to explore the limits of the prototype (e.g., limited count rate performance) and, more importantly, its potential to image freely moving subjects. Several more concepts have been proposed to improve the PET performance, reduce the scanner weight, increase the subject comfort, and allow more mobility during the examination (28).

Another wearable concept, called Mind-Tracker PET, was recently proposed (Fig. $1 \mathrm{H}$ ). It consisted of 16 modules positioned $20 \mathrm{~cm}$ apart, each with a $10 \times 10$ array of $3 \times 3 \times 20 \mathrm{~mm}^{3}$ LYSO crystals read out by a $10 \times 10$ SensL SiPM array (Fig. $2 \mathrm{H}$ ). With a weight of about $3 \mathrm{~kg}$, the Mind-Tracker "is lighter than the commonly-used motorcycle helmet," which would allow imaging of subjects in a vertical position for extended periods (29).

\section{Unconventional Scanner Geometry Concepts}

Several unconventional scanner geometries have been suggested to further increase the sensitivity. For example, the detectors were arranged in a compact hemisphere, truly resembling a helmet, which improved the solid-angle coverage at the edge of the FOV (where most cortical brain structures are located), and add-on detectors were placed in various locations (i.e., chin, ear, neck) to also increase the sensitivity at the center of the hemisphere (where the cerebellum is located) $(30,31)$. Compared with the conventional cylindrical geometry, the sensitivity was improved $50 \%$ for the helmet geometry. More significantly, the add-on detectors increased the sensitivity in a region of interest at the center by $200 \%$, which is remarkable considering that only $12 \%$ more detector 
material was used (31). Ahmed et al. also performed simulations to compare the performance of the helmet-chin geometry to that of a cylindrical scanner using the same number of detectors. In addition to 1.5 times higher sensitivity, they also reported more than a $40 \%$ increase in peak noise-equivalent count rate (32).

A helmet design was also investigated for developing a nextgeneration ambulatory microdose PET scanner (Fig. 3A). Instead of a hemispheric arrangement, the authors proposed using a top panel (with 52 detector blocks), 6 side rings (with 38, 38, 36, 33, 29 , and 26 blocks), and a bottom panel (with $4 \times 4$ blocks). The Monte Carlo simulations showed the sensitivity for this configuration to be 4.2 times higher than that of the cylindrical brain PET scanner. Furthermore, the authors showed that the DOI information is essential for quantifying uptake in small regions using a scanner with a compact geometry and that improving the time-offlight performance from 400- to 200-ps results in better image quality (33).

But can an even higher sensitivity be achieved? Shi et al. investigated several polyhedron geometries and concluded that the dodecahedron design provides the same solid-angle coverage as a spherical cap, both substantially higher than that of a $30-\mathrm{cm}-$ diameter cylindrical scanner. They also claimed that the dodecahedral geometry provides the best tradeoff between solid-angle coverage and reasonable complexity to allow manufacturing and even proposed a light-sharing scheme for reading out the pentagon-shaped detectors using photomultiplier tubes (34). Subsequently, they performed careful Monte Carlo simulations for the dodecahedral geometry (Fig. 3B). The scanner they simulated consisted of 11 pentagon-shaped DOI detector modules, with each two opposing modules being $30 \mathrm{~cm}$ apart. The simulations showed that dodecahedral PET could produce lower-noise images than the HRRT, consistent with the sensitivity improvement $(72 \%$ and 4.91 times better than the helmet design and HRRT, respectively). Assuming a 6.67-mm DOI resolution, the estimated spatial resolution at the center of the FOV was $1.98 \mathrm{~mm}$ (35).

The concept of a $4 \pi$ solid angle has intrigued researchers from the early days of PET as a means to improve the sensitivity of firstgeneration scanners and led to the development of image reconstruction algorithms suitable for spherical geometries more than 4 decades ago $(36,37)$. Cho et al. proposed a spherical geometry with the goal of almost $4 \pi$ detection of photons from the central region of the subject. Although the brain was not the target organ in that study, the authors performed a careful analysis of the potential performance of such a scanner and showed that its sensitivity could be 3 times higher than that of a cylindrical scanner (38).

More recently, Moghaddam et al. designed a spherical PET scanner using liquid xenon instead of a solid scintillator material

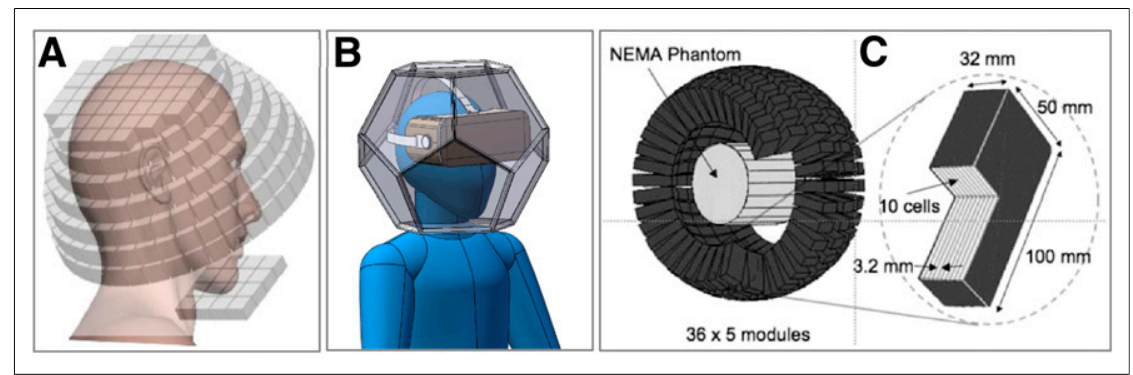

FIGURE 3. Examples of dedicated PET scanners with unconventional geometries: Helmet-PET plus chin (33) (A), dodecahedral (courtesy of Qiyu Peng, Lawrence Berkeley National Laboratory) (B), and spherical (39) (C). NEMA = National Electrical Manufacturers Association. as the detector (Fig. 3C). The estimated sensitivity was $1.14 \%$ for 100-mm-thick detectors (39).

Schmidtlein et al. proposed a spherical brain PET scanner using autonomous detector arrays with integrated signal processing electronics stacked together to form autonomous detector blocks. The authors argued this configuration leads to substantial performance improvements $(>40 \%$ better count rate performance than cylindrical geometry), lower weight, less complexity, and overall higher flexibility in terms of the scanner design (40).

\section{MR-COMPATIBLE PET INSERTS}

\section{Conventional Scanner Geometry Devices}

The first integrated PET/MRI scanner for human use, called BrainPET, was designed and built by Siemens (41). This prototype PET insert was integrated with the standard 3-T MRI scanner (Fig. 4). The PET detector module consisted of a $12 \times 12$ array of $2.5 \times$ $2.5 \times 20 \mathrm{~mm}^{3}$ lutetium oxyorthosilicate crystals read out by a $3 \times 3$ array of avalanche photodiodes (Hamamatsu). Thirty-two individually shielded detector cassettes (each consisting of 6 detector modules) made up the PET gantry, with an inner diameter of 35 $\mathrm{cm}$ and an axial FOV of $19.125 \mathrm{~cm}$ (Fig. 5A). The spatial resolution of the BrainPET was below $3 \mathrm{~mm}$ at $1 \mathrm{~cm}$ off-center but degraded to about $6 \mathrm{~mm}$ at $10 \mathrm{~cm}$ off-center because of the lack of DOI information. The sensitivity was $7.2 \%$ at the center of the FOV (42). The BrainPET prototypes installed at 4 centers in Germany and the United States have been used in a myriad of studies: to investigate the mutual interference between the two devices and the performance of the PET camera (42), to develop methods to use the information obtained from one device to improve the other (43-47), and to perform proof-of-principle studies on small-animal, nonhuman primates and humans (48-53).

To minimize the mutual interference with the 3-T MRI scanner, Jung et al. from Korea used charge signal transmission. Specifically, they sent the outputs of the detectors to preamplifiers using 4-m-long flat, flexible electrical cables shielded with a 0.24 -mm-thick aluminum sheet. Additionally, they used 4-side tileable arrays to extend the axial FOV. The detector module had a $4 \times 4$ array of LYSO crystals $\left(3 \times 3 \times 20 \mathrm{~mm}^{3}\right)$ coupled to a $4 \times 4$ array of Geiger-mode avalanche photodiodes. The detector block consisted of $4 \times 4$ arrays of detector modules. Eighteen of these blocks circularly mounted made up the gantry, with an inner diameter of $390 \mathrm{~mm}$ and an axial length of $60 \mathrm{~mm}$ (Fig. 5B). Sensitivity of about $0.8 \%$ and a $3-\mathrm{mm}$ spatial resolution were measured at the center of the FOV, and no performance degradation was reported inside the MR scanner (54).

To improve sensitivity without sacrificing the spatial resolution uniformity across the FOV and maintaining the MR compatibility, Nishikido et al. from Japan proposed an add-on PET insert with 4-layer DOI-capable detectors read out by MPPCs (55). A first-generation prototype detector module consisted of 4 layers of $12 \times 4$ lutetium gadolinium orthosilicate scintillator crystals $\left(2.9 \times 2.9 \times 5 \mathrm{~mm}^{3}\right)$ with reflector arranged in a way that allowed the separation of all the crystals in each of the layers as described previously (56). Subsequently, the LYSO crystal dimensions were reduced to $2 \times 2 \times 5 \mathrm{~mm}^{3}$, and they were arranged in 4 layers of $19 \times 6$ arrays (Fig. 5C) (57). The PET detector modules were mounted 


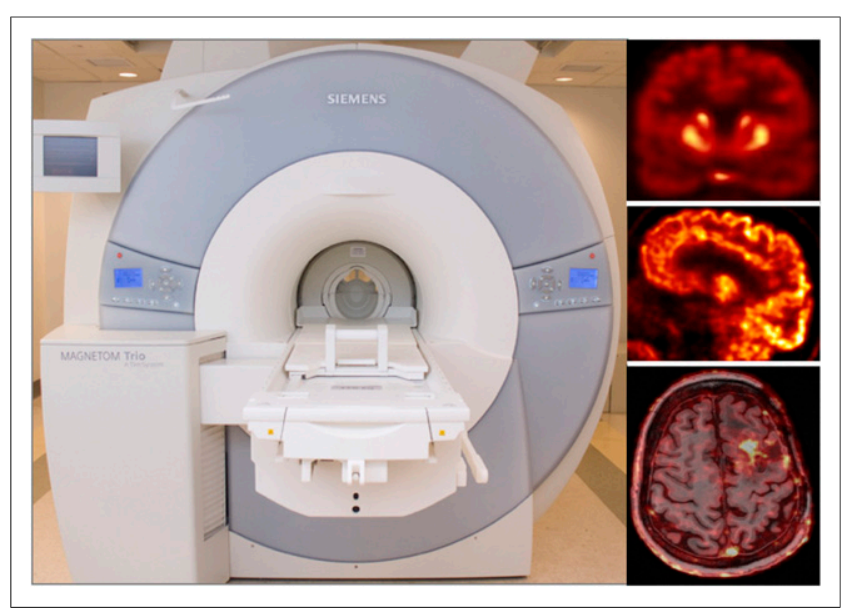

FIGURE 4. BrainPET prototype installed inside 3-T MR scanner and representative images from studies performed at Athinoula $A$. Martinos Center using ${ }^{11} \mathrm{C}-\mathrm{NNC} 112$ (coronal view [top]), ${ }^{18} \mathrm{~F}-\mathrm{FDG}$ (sagittal view [middle]), and ${ }^{11} \mathrm{C}$-temozolomide (transaxial view and fused with morphologic MR image [bottom]).

between the elements of a 3-T MRI birdcage coil to evaluate their performance. No degradation in crystal identification and energy resolution was noted inside the MRI scanner. The spatial resolution ranged from $2.3 \mathrm{~mm}$ at the center of the FOV to $3.5 \mathrm{~mm}$ offcenter. The sensitivity was not estimated given the relatively limited extent of the axial FOV $(1.2 \mathrm{~cm})$, which would not be sufficient for actual brain studies, as acknowledged by the authors (57).

A different approach to minimizing the interference between the two modalities was proposed by researchers from Stanford. They developed a radiofrequency-penetrable PET insert by having the PET ring electrically float relative to the MRI system and leaving small 1-mm air gaps between adjacent PET detector module shields (58). Each detector module consisted of a $2 \times 4$ array of $4 \times 4$ arrays of LYSO crystals $\left(3.2 \times 3.2 \times 20 \mathrm{~mm}^{3}\right)$ coupled to SiPM arrays (SensL). The inner diameter of the ring was $32 \mathrm{~cm}$, and the axial FOV was $2.6 \mathrm{~cm}$ (Fig. 5D). Instead of using coaxial cables, the authors used optical fibers to transmit the analog PET output signals to the electronics located outside the MR. Additionally, they used nonmagnetic batteries to power the PET insert. These modifications allowed them to isolate the electrical grounds of the two systems, further minimizing the potential for mutual interference. The MR body coil was used to transmit and receive the radiofrequency signal through the gaps between the modules. This allowed the simultaneous PET/MRI data acquisition without the need for building dedicated radiofrequency coils, although substantially improved MR performance was recently demonstrated when positioning the receive coil inside the insert (59). Additionally, the radiofrequency penetrability could be improved by optimizing the geometry of the PET insert (e.g., using a rectangular vs. trapezoidal shield box and increasing the interdetector gap to $3 \mathrm{~mm}$ ) (60).

The goal of the Multimodal Imaging of Neurologic Disorders View (MINDView) project was to develop a high-spatial-resolution and high-sensitivity scanner to visualize neurotransmitter pathways and their disruption in psychiatric disorders (61). More specifically, the requirements for the spatial resolution were to be approximately $1 \mathrm{~mm}$ in the center of the brain and relatively uniform across the FOV. Two crystal configurations were investigated for achieving these goals: a 3 -layer staggered pixelated array $(35 \times 35$ array of
$1.5 \times 1.5 \times 6 \mathrm{~mm}^{3}$ LYSO crystals $)$ and a monolithic $(50 \times 50 \times$ $20 \mathrm{~mm}^{3}$ LYSO) block, the latter being the one expected to provide the best overall performance at a reasonable cost. Similarly, two approaches for the readout electronics were investigated, and the one selected used a special charge division network that provided information for each row and column output of the SiPM array. To minimize claustrophobia, the authors investigated an open design for the radiofrequency coil and showed that the coil rungs and the PET shield surface must be separated by at least $30 \mathrm{~mm}$. Decreasing it to $20 \mathrm{~mm}$ reduced the B1 field strength by about $50 \%$. A scanner consisting of 60 detector modules arranged in 3 rings with a diameter of $33 \mathrm{~cm}$ and a total axial coverage of $15.2 \mathrm{~cm}$ (Fig. 5E) was recently built (62). The 1.6-mm hot rods could be visualized in the Derenzolike phantom images. The scanner sensitivity was $2.7 \%$ at the center of the FOV. The PET scanner was also tested inside a Biograph mMR scanner (Siemens Healthineers), and no significance interference was observed. MR images of a patient were also successfully acquired with a dummy version of the PET device in place.

The TRIMAGE project was another ambitious effort funded by the European Union aimed at developing a cost-effective technology that allows the simultaneous acquisition of PET/MRI and electroencephalography data (63). As opposed to all the other approaches discussed above, the MR component of this trimodal system was based on a novel compact 1.5-T noncryogenic magnet. The PET scanner, with an inner diameter of $260 \mathrm{~mm}$ and an axial FOV of $160 \mathrm{~mm}$, consisted of $18 \mathrm{LYSO} / \mathrm{SiPM}$ detectors, each made up of 3 modules, divided into 4 submodules, called tiles. Each tile had 2 half-pitch staggered crystal layers: the top with $7 \times$ 7 crystals of $3.3 \times 3.3 \times 8 \mathrm{~mm}^{3}$ and the bottom with $8 \times 8$ crystals of $3.3 \times 3.3 \times 12 \mathrm{~mm}^{3}$ (Fig. $5 \mathrm{~F}$ ). The photodetector consisted of 2 matrices of $8 \times 4$ near-ultraviolet SiPMs (AdvanSiD). A

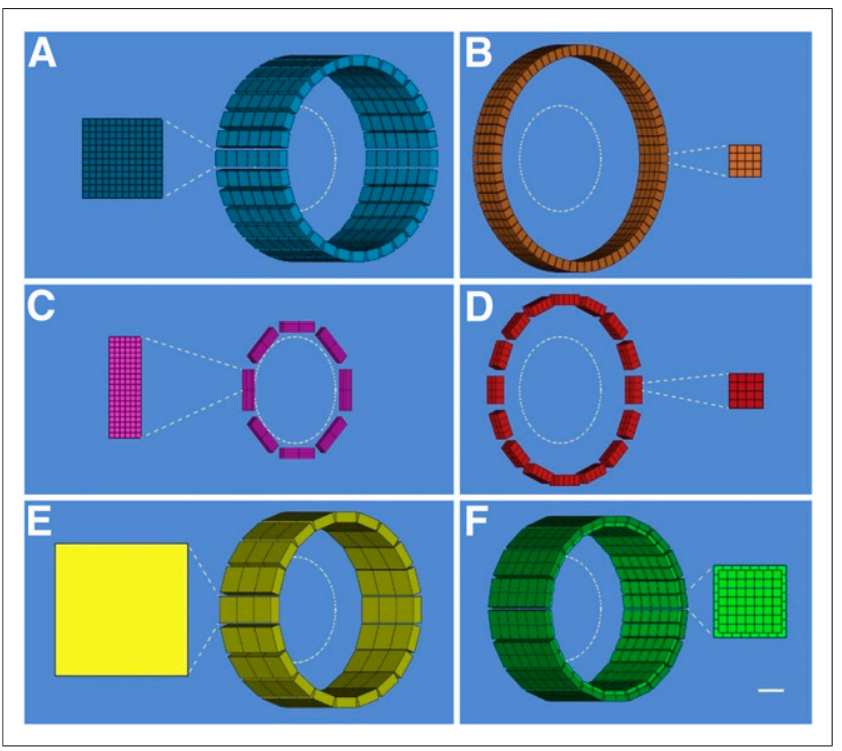

FIGURE 5. Schematic drawings (angled view) showing configuration of detector modules and cross-sectional detailed views (scale, 5:1) of scintillator arrays for dedicated MR-compatible PET inserts with conventional geometries: BrainPET (A), charge signal transmission (B), add-on PET insert with 4-layer DOI-capable detectors (C), radiofrequencypenetrable PET (D), MINDView (E), and TRIMAGE (F). A 20-cm-diameter circle is shown inside each scanner. Scale bar represents $1 \mathrm{~cm}$ in detailed views. 
64-channel MR-compatible commercially available electroencephalography system was used (Brain Products). Pilot studies were performed to assess the mutual interference between the 3 modalities and to optimize the data acquisition and processing protocols. For example, the impact of the electroencephalography cap and PET data quantification was assessed using the 3-T MRBrainPET scanner (64). Simultaneous trimodal PET/MR/electroencephalography measurements were also performed on healthy volunteers using the same device $(65,66)$.

\section{Unconventional Scanner Geometry Concept}

At the Athinoula A. Martinos Center, we have recently started to develop and evaluate the PET detector module and system technology for a next-generation 7-T MR-compatible PET insert with dramatically improved sensitivity. A possible spherical geometry that would maximize the solid-angle coverage is shown in Figure 6. In this case, the PET detectors with DOI capabilities are arranged around a partial sphere (32-cm inner diameter) with a 25-cm-diameter front opening to allow the positioning of the subject (wearing MR-compatible goggles to maximize comfort and display stimuli) and a 9-cm-diameter back opening for the cables. The estimated solid-angle coverage for this configuration is about $71 \%$. Assuming $90 \% 511-\mathrm{keV}$ photon detection efficiency (for 2.6-cmlong lutetium oxyorthosilicate crystals) and $40 \%$ scatter fraction, this translates into an approximately $25 \%$ sensitivity for detecting true coincidences. Furthermore, when the virtual sensitivity amplifier of including the time-of-flight information is considered (67), the effective sensitivity of this scanner could be as high as 50\%, a dramatic improvement compared with current values.

\section{ASPIRATIONAL DESIGN CRITERIA FOR DEDICATED PET BRAIN SCANNERS}

Although the performance of a PET scanner will always depend on the available technology, profitability, and manufacturing practicality requirements; the often limited resources available to developers in academia; and many other considerations, it might be useful to discuss the aspirational criteria for future systems, focusing on spatial resolution, sensitivity, and the need for multimodal

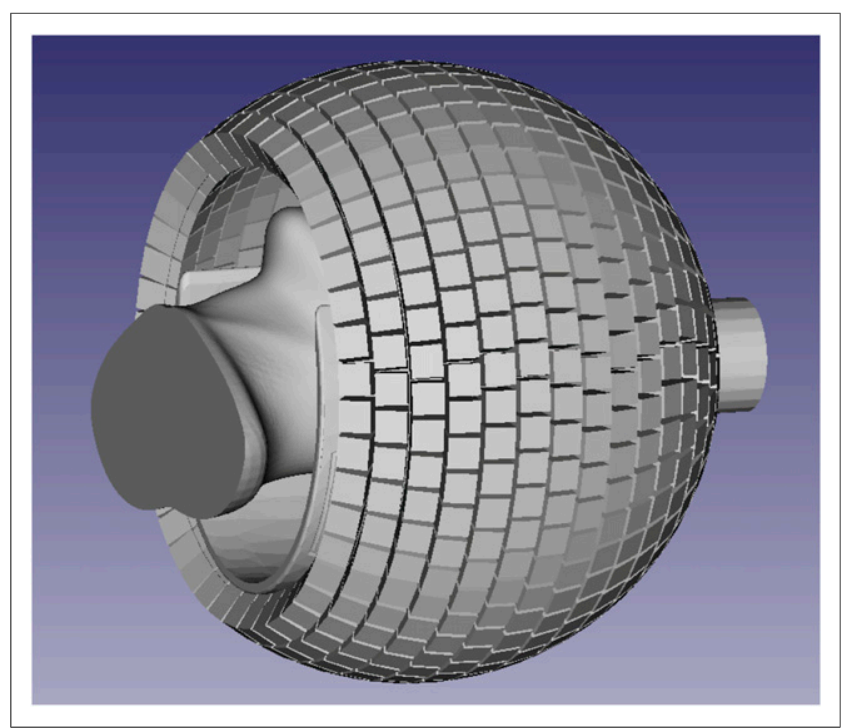

FIGURE 6. Possible geometry for 7-T MRI-compatible high-sensitivity brain PET scanner. integration. In each case, the need for improvement will be briefly considered before the difficult and perilous task of predicting the future is taken on.

Improving the spatial resolution is perhaps the easiest to justify as this is often singled out as one of the main limitations of PET. Indeed, partial-volume effects bias the PET estimates in cortical structures in virtually all neurologic studies performed today. Furthermore, there is growing interest in imaging even smaller structures such as hippocampal subfields and thalamic and brain stem nuclei. Future-generation scanners should aspire to reach the fundamental limit placed by physics (positron range and noncollinearity), which is $0.7 \mathrm{~mm}$ for a dedicated brain PET scanner (assuming a 20-cm-diameter scanner, perfect detectors with no decoding error, DOI capability, and ${ }^{18} \mathrm{~F}$-labeled radiotracers). The contribution of the positron range error could be further reduced inside a high-field magnet. Additionally, the apparent spatial resolution could be further improved by incorporating information from higher-resolution MR images and minimizing the impact of head motion using the simultaneously acquired MR data (44).

When it comes to setting sensitivity targets, higher values will always be desired because PET is a counting-statistics-limited technique and recording a larger fraction of the true coincidences leads to better image quality, shorter acquisition time, lower radiation exposure, and higher temporal resolution or the ability to detect smaller changes in radiotracer concentration. Although the relevance of the first 3 choices is obvious, the last 2 need further discussion. The highest temporal resolution reported to date is approximately $1 \mathrm{~min}(68)$, but frames on the order of 5-10 $\mathrm{min}$ are most often used in research studies, whereas data collected over tens of minutes are averaged for routine clinical studies. Thus, traditional neurochemical dynamic measurements made using PET have, for most of its history, provided a temporal snapshot of a single point in time, as opposed to reflecting the dynamics of the receptor systems themselves. However, we now know that phasic neurotransmitters such as dopamine release connected to cognitive activity or psychostimulants can be modulated within time scales on the order of seconds $(69,70)$ and that this burst firing of dopamine receptors on these time scales has been demonstrated, through experiments and modeling, to be the likely carrier of meaningful information on the firing activities of dopamine neurons (71). The ability to detect very small changes in radiotracer concentration is necessary because of the long time constants of the PET tracer kinetics themselves, leading to only small changes in observed signal over short periods. This ability depends on the available signal-to-noise ratio, which in the case of PET represents the ratio of true coincidences to background events. The situation is analogous to the situation in functional MRI, where the well-known hemodynamic response function evolves over many seconds. Yet with superior sensitivity, investigators have now observed neural oscillatory behavior of almost 1 $\mathrm{Hz}$ (72), well beyond initial expectations. Using PET scanner geometries that maximize the solid-angle coverage and highperformance detectors, the sensitivity could be upward of $25 \%$ (or upward of 50\% when including the time-of-flight sensitivity amplifier effect), which is likely close to the practical limit (short of surrounding the subject with detectors). Of course, such improvements in sensitivity will require similar enhancements in the photon detection performance (perhaps novel scintillator materials, more efficient photon detectors, faster readout electronics, and other improvements) to support the processing of the expected 
very high event rates. Furthermore, recent studies suggest that the apparent sensitivity of our measurements can be increased for specific applications using machine learning approaches (73). In fact, future-generation dedicated brain PET scanners will likely combine hardware and machine learning developments to continue to push the apparent sensitivity limits (74).

Finally, although the marriage of PET and MRI seemed a match made in heaven (75), the multimodal integration should not stop with these 2 technologies. For example, integrating dynamic PET measurements with functional MRI and electroencephalography observations would enable fundamentally new types of studies of human brain network function, as it would allow neuromodulatory dynamics to be linked directly with large-scale brain network activity and fast neurophysiologic signals. Similarly, integrating optical imaging techniques could provide additional information about the hemodynamic response dynamics (76) or a more comprehensive understanding of the physiology of the functional connectivity signal (77).

\section{THE STATUS OF DEDICATED BRAIN PET INSTRUMENTATION AND FUTURE PERSPECTIVES}

Several of the brain PET scanners developed to date have demonstrated substantially improved performance characteristics compared with their whole-body counterparts (albeit still far from the goals discussed above). Furthermore, the more versatile designs proposed allow novel types of neuroimaging studies (e.g., scanning subjects in new settings). Given these advantages and the urgent need to address the huge burden from neuropsychiatric disorders, one would expect the demand for these specialized devices to be high. However, most of the scanners described above were developed in academic centers and were not commercialized. Even the HRRT, arguably the most successful of them, has been installed at only a limited number of sites and is no longer commercially available.

There are several possible explanations for why this market has not matured as quickly as initially expected. First, from an economic point of view, the equipment manufacturers and potential users have been reluctant to develop and invest in an organ-specific device, especially since PET is already an expensive imaging modality because of the radiochemistry-related costs. This is slowly changing as novel detector technologies, combined with more compact designs, allow substantial cost savings without performance degradation and thus the development of substantially lowercost devices. Even an MR-compatible PET insert can be viewed as a cost-effective way of converting an already existing MRI scanner into a hybrid device, particularly for neuroimaging-focused centers. Second, from a clinical applications perspective, the demand for dedicated brain PET scanners has been quite low, likely because not enough of the many research breakthroughs in this field have been successfully translated to the clinic. In fact, whole-body oncology has been the main driver of technologic developments in the PET world for 3 decades. The use of PET for imaging neurodegeneration has continuously grown in recent years, and this application has the potential to begin to restore the balance. Additionally, nextgeneration very high-performance devices could allow PET to expand in novel directions such as imaging the dynamics of neurotransmitter function at high temporal resolution or metabolic processes/protein accumulations down to extremely low (pico- to nanomolar) concentrations, which could enable early diagnosis and direct monitoring of drug effects. Another factor that has slowed progress in dedicated brain PET instrumentation was the introduction of functional MRI (78), an imaging modality that does not involve ionizing radiation and allows the assessment of brain activation more quickly, less expensively, and less invasively. Nevertheless, despite continued progress in functional MRI techniques and hardware over the last almost 3 decades, this imaging modality still cannot compete with PET for providing direct information about neurotransmitter function. The availability of integrated PET/MRI scanners that allow the simultaneous assessment of molecular and functional changes in the brain will hopefully give neuroscientists the opportunity to discover or rediscover PET and its unique capabilities. Finally, the recent development of machine learning techniques aimed at artificially increasing the performance of PET (73) could again shift the focus from further improving the dedicated brain PET hardware, because similar information could in principle be obtained using artificial-intelligence-enabled lowercost PET hardware in the case of very clearly defined applications and assuming enough training data are available. However, the more likely scenario is that these techniques will instead be used for addressing some of the limitations of PET (e.g., further improve performance and reduce the acquisition time or radiation exposure). Moreover, combining artificial intelligence and novel high-performance hardware approaches could lead to dramatic improvements in PET performance (74).

\section{CONCLUSION}

Substantial progress has been made in the field of dedicated brain PET instrumentation. However, sustained effort is still needed to move from promising concepts to specialized products that can broaden our understanding of the human brain and, ultimately, address the many unmet clinical needs in neurology and psychiatry.

\section{DISCLOSURE}

This work was supported in part by the NIH BRAIN Initiative (1R01 EB026995). No other potential conflict of interest relevant to this article was reported.

\section{ACKNOWLEDGMENTS}

I would like to thank Dr. Bruce Rosen for the many insightful discussions about current and future applications of multimodal brain imaging technology.

\section{REFERENCES}

1. Jones T, Rabiner EA. The development, past achievements, and future directions of brain PET. J Cereb Blood Flow Metab. 2012;32:1426-1454.

2. Jones T, Townsend D. History and future technical innovation in positron emission tomography. J Med Imaging (Bellingham). 2017;4:011013.

3. Thompson CJ, Yamamoto YL, Meyer E, Positome II: a high efficiency positron imaging device for dynamic brain studies. IEEE Trans Nucl Sci. 1979;26:583589

4. Hoffman EJ, Phelps ME, Huang SC. Performance evaluation of a positron tomograph designed for brain imaging. J Nucl Med. 1983;24:245-257.

5. Freifelder R, Karp JS, Geagan M, Muehllehner G. Design and performance of the HEAD PENN-PET scanner. IEEE Trans Nucl Sci. 1994;41:1436-1440.

6. Moses WW, Virador PRG, Derenzo SE, Huesman RH, Budinger TF. Design of a high-resolution, high-sensitivity PET camera for human brains and small animals. IEEE Trans Nucl Sci. 1997;44:1487-1491.

7. Watanabe M, Shimizu K, Omura T, et al. A new high-resolution PET scanner dedicated to brain research. IEEE Trans Nucl Sci. 2002;49:634-639.

8. Karp JS, Surti S, Daube-Witherspoon ME, et al. Performance of a brain PET camera based on Anger-logic gadolinium oxyorthosilicate detectors. J Nucl Med. 2003;44:1340-1349. 
9. Rowland DJ, Cherry SR. Small-animal preclinical nuclear medicine instrumentation and methodology. Semin Nucl Med. 2008;38:209-222.

10. Budinger TF. PET instrumentation: what are the limits? Semin Nucl Med. 1998;28:247-267.

11. Schmand M, Eriksson L, Casey ME, et al. Performance results of a new DOI detector block for a high resolution PET-LSO research tomograph HRRT. IEEE Trans Nucl Sci. 1998;45:3000-3006.

12. Wienhard K, Schmand M, Casey ME, et al. The ECAT HRRT: performance and first clinical application of the new high resolution research tomograph. IEEE Trans Nucl Sci. 2002;49:104-110.

13. Eggers C, Hilker R, Burghaus L, Schumacher B, Heiss WD. High resolution positron emission tomography demonstrates basal ganglia dysfunction in early Parkinson's disease. J Neurol Sci. 2009;276:27-30.

14. Bishu S, Schmidt KC, Burlin T, et al. Regional rates of cerebral protein synthesis measured with $1-\left[1-{ }^{11} \mathrm{C}\right]$ leucine and PET in conscious, young adult men: normal values, variability, and reproducibility. J Cereb Blood Flow Metab. 2008;28: $1502-1513$.

15. Cho Z-H, Son Y-D, Kim H-K, et al. Observation of glucose metabolism in the thalamic nuclei by fusion PET/MRI. J Nucl Med. 2011;52:401-404.

16. Rahmim A, Bloomfield P, Houle S, et al. Motion compensation in histogrammode and list-mode EM reconstructions: beyond the event-driven approach. IEEE Trans Nucl Sci. 2004;51:2588-2596.

17. Boellaard R, Knaapen P, Rijbroek A, Luurtsema GJJ, Lammertsma AA. Evaluation of basis function and linear least squares methods for generating parametric blood flow images using ${ }^{15} \mathrm{O}$-water and positron emission tomography. $\mathrm{Mol}$ Imaging Biol. 2005;7:273-285.

18. Ding Y-S, Singhal T, Planeta-Wilson B, et al. PET imaging of the effects of age and cocaine on the norepinephrine transporter in the human brain using $(\mathrm{S}, \mathrm{S})$ $\left[{ }^{11} \mathrm{C}\right] \mathrm{O}-\mathrm{methylreboxetine}$ and HRRT. Synapse. 2010;64:30-38.

19. Yamaya T, Hagiwara N, Obi T, et al. Transaxial system models for jPET-D4 image reconstruction. Phys Med Biol. 2005;50:5339-5355.

20. Yamaya T, Yoshida E, Kitamura K, et al. First human brain images of the jPETD4 using 3D OS-EM with a pre-computed system matrix. In: 2006 IEEE Nuclear Science Symposium Conference Record. Piscataway, NJ: IEEE; 2006:33843387.

21. Worstell W, Adler S, Domigan P, et al. Dynamic brain imaging with low injection dose using the NeuroPET [abstract]. J Nucl Med. 2009;50(suppl 2):527.

22. Grogg KS, Toole T, Ouyang J, et al. National Electrical Manufacturers Association and clinical evaluation of a novel brain PET/CT scanner. J Nucl Med. 2016;57:646-652.

23. Watanabe M, Saito A, Isobe T, et al. Performance evaluation of a high-resolution brain PET scanner using four-layer MPPC DOI detectors. Phys Med Biol. 2017;62:7148-7166.

24. Yamamoto S, Honda M, Oohashi T, Shimizu K, Senda M. Development of a brain PET system, PET-Hat: a wearable PET system for brain research. IEEE Trans Nucl Sci. 2011;58:668-673.

25. Majewski S, Proffitt J, Brefczynski-Lewis J, et al. HelmetPET: a silicon photomultiplier based wearable brain imager. In: 2011 IEEE Nuclear Science Symposium Conference Record. Piscataway, NJ: IEEE; 2011:4030-4034.

26. Kinahan P, Majewski S, Elston B, et al. Design considerations for AMPET: the ambulatory micro-dose, wearable PET brain imager [abstract]. J Nucl Med. 2015;56(suppl 3):1540.

27. Bauer CE, Brefczynski-Lewis J, Marano G, et al. Concept of an upright wearable positron emission tomography imager in humans. Brain Behav. 2016;6: e00530.

28. Melroy S, Bauer C, McHugh M, et al. Development and design of next-generation head-mounted ambulatory microdose positron-emission tomography (AMPET) system. Sensors (Basel). 2017;17:E1164.

29. Xu J, Zhao Z, Xie S, Shi D, Huang Q, Peng Q. Mind-Tracker PET: a wearable PET camera for brain imaging. IEEE Xplore website. https://ieeexplore.ieee.org/ document/8532744. Published 2017. Accessed May 7, 2019.

30. Tashima H, Ito $\mathrm{H}$, Yamaya $\mathrm{T}$. A proposed helmet-PET with a jaw detector enabling high-sensitivity brain imaging. IEEE Xplore website. https://ieeexplore. ieee.org/document/6829074. Published 2013. Accessed May 7, 2019.

31. Tashima H, Yamaya T. Proposed helmet PET geometries with add-on detectors for high sensitivity brain imaging. Phys Med Biol. 2016;61:7205-7220.

32. Ahmed AM, Tashima H, Yoshida E, Nishikido F, Yamaya T. Simulation study comparing the helmet-chin PET with a cylindrical PET of the same number of detectors. Phys Med Biol. 2017;62:4541-4550.

33. Gong K, Majewski S, Kinahan PE, et al. Designing a compact high performance brain PET scanner: simulation study. Phys Med Biol. 2016;61:3681-3697.

34. Shi H, Du D, Xu J, Su Z, Peng Q. Design study of dedicated brain PET with polyhedron geometry. Technol Health Care. 2015;23(suppl):S615-S623.
35. Tao W, Weng F, Zan Y, et al. Simulation study of a dedicated brain PET system with dodecahedral geometry. Med Phys. 2018;45:3297-3304.

36. Pelc NJ, Chesler DA. Utilization of cross-plane rays for three-dimensional reconstruction by filtered back-projection. J Comput Assist Tomogr. 1979;3:385395.

37. Ra JB, Lim CB, Cho ZH, Hilal SK, Correll J. A true three-dimensional reconstruction algorithm for the spherical positron emission tomograph. Phys Med Biol. 1982;27:37-50.

38. Cho ZH, Hong KS, Hilal SK. Spherical positron emission tomograph (SPET) I: performance analysis. Nucl Instrum Methods Phys Res. 1984;225: 422-438.

39. Moghaddam NM, Karimian A, Mostajaboddavati SM, Vondervoort E, Sossi V. Preliminary design and simulation of a spherical brain PET system (SBPET) with liquid xenon as scintillator. Nukleonika. 2009;54:33-38.

40. Schmidtlein CR, Turner JN, Thompson MO, et al. Initial performance studies of a wearable brain positron emission tomography camera based on autonomous thin-film digital Geiger avalanche photodiode arrays. J Med Imaging (Bellingham). 2017;4:011003.

41. Schlemmer H-PW, Pichler BJ, Schmand M, et al. Simultaneous MR/PET imaging of the human brain: feasibility study. Radiology. 2008;248:1028-1035.

42. Kolb A, Wehrl HF, Hofmann M, et al. Technical performance evaluation of a human brain PET/MRI system. Eur Radiol. 2012;22:1776-1788.

43. Catana C, Benner T, Van Der Kouwe A, et al. MRI-assisted PET motion correction for neurologic studies in an integrated MR-PET scanner. J Nucl Med. 2011;52:154-161.

44. Chen KT, Salcedo S, Gong K, et al. An efficient approach to perform MRassisted PET data optimization in simultaneous PET/MR neuroimaging studies. J Nucl Med. June 22, 2018 [Epub ahead of print].

45. Izquierdo-Garcia D, Hansen AE, Förster S, et al. An SPM8-based approach for attenuation correction combining segmentation and nonrigid template formation: application to simultaneous PET/MR brain imaging. J Nucl Med. 2014;55:18251830 .

46. Scheins J, Weirich C, Caldeira L, et al. High-resolution, quantitative 3D PET image reconstruction for the Siemens hybrid 3T MR/BrainPET scanner using the PET reconstruction software toolkit (PRESTO) [abstract]. EJNMMI Phys. 2014;1(suppl 1):A51.

47. da Silva NA, Caldeira L, Herzog H, et al. Automatic derivation of an MR-PET image-based input function for quantification of ${ }^{18} \mathrm{~F}$-FET. EJNMMI Phys. 2015;2:A27.

48. Alshikho MJ, Zürcher NR, Loggia ML, et al. Glial activation colocalizes with structural abnormalities in amyotrophic lateral sclerosis. Neurology. 2016;87: 2554-2561.

49. Atzil S, Touroutoglou A, Rudy T, et al. Dopamine in the medial amygdala network mediates human bonding. Proc Natl Acad Sci USA. 2017;114:23612366.

50. Roffman JL, Tanner AS, Eryilmaz H, et al. Dopamine D1signaling organizes network dynamics underlying working memory. Sci Adv. 2016;2:e1501672.

51. Loggia ML, Chonde DB, Akeju O, et al. Evidence for brain glial activation in chronic pain patients. Brain. 2015;138:604-615.

52. Lohmann P, Herzog H, Rota Kops E, et al. Dual-time-point O-(2-[ ${ }^{18}$ F $]$ fluoroethyl)-L-tyrosine PET for grading of cerebral gliomas. Eur Radiol. 2015;25: 3017-3024.

53. Sander CY, Hooker JM, Catana C, et al. Neurovascular coupling to D2/D3 dopamine receptor occupancy using simultaneous PET/functional MRI. Proc Natl Acad Sci USA. 2013;110:11169-11174.

54. Jung JH, Choi Y, Jung J, et al. Development of PET/MRI with insertable PET for simultaneous PET and MR imaging of human brain. Med Phys. 2015;42:23542363.

55. Nishikido F, Obata T, Shimizu K, et al. Feasibility of a brain-dedicated PET-MRI system using four-layer DOI detectors integrated with an RF head coil. Nucl Instrum Methods Phys Res A. 2014;756:6-13.

56. Tsuda T, Murayama H, Kitamura K, et al. A four-layer depth of interaction detector block for small animal PET. IEEE Trans Nucl Sci. 2004;51:25372542 .

57. Nishikido F, Fujiwara M, Tashima H, et al. Development of a full-ring "add-on PET" prototype: a head coil with DOI-PET detectors for integrated PET/MRI. Nucl Instrum Methods Phys Res A. 2017;863:55-61.

58. Grant AM, Lee BJ, Chang CM, Levin CS. Simultaneous PET/MR imaging with a radio frequency-penetrable PET insert. Med Phys. 2017;44:112-120.

59. Lee BJ, Watkins RD, Lee KS, Chang C-M, Levin CS. Performance evaluation of RF coils integrated with an RF-penetrable PET insert for simultaneous PET/ MRI. Magn Reson Med. 2019;81:1434-1446. 
60. Akram MSH, Levin CS, Obata T, Hirumi G, Yamaya T. Geometry optimization of electrically floating PET inserts for improved RF penetration for a 3 T MRI system. Med Phys. 2018;45:4627-4641.

61. González AJ, Majewski S, Sánchez F, et al. The MINDView brain PET detector, feasibility study based on SiPM arrays. Nucl Instrum Methods Phys Res A. 2016;818: 82-90.

62. Benlloch JM, González AJ, Pani R, et al. The MINDVIEW project: first results. Eur Psychiatry. 2018;50:21-27.

63. Del Guerra A, Ahmad S, Avram M, et al. TRIMAGE: a dedicated trimodality (PET/MR/EEG) imaging tool for schizophrenia. Eur Psychiatry. 2018;50:7-20.

64. Rajkumar R, Rota Kops E, Mauler J, et al. Simultaneous trimodal PET-MR-EEG imaging: do EEG caps generate artefacts in PET images? PLoS One. 2017;12: e0184743.

65. Shah NJ, Arrubla J, Rajkumar R, Farrher E, Mauler J, Kops ER. Multimodal fingerprints of resting state networks as assessed by simultaneous trimodal MRPET-EEG imaging. Sci Rep. 2017;7:6452.

66. Rajkumar R, Farrher E, Mauler J, et al. Comparison of EEG microstates with resting state fMRI and FDG-PET measures in the default mode network via simultaneously recorded trimodal (PET/MR/EEG) data. Hum Brain Mapp. October 27, 2018 [Epub ahead of print].

67. Conti M. Focus on time-of-flight PET: the benefits of improved time resolution. Eur J Nucl Med Mol Imaging. 2011;38:1147-1157.

68. Constantinescu CC, Bouman C, Morris ED. Nonparametric extraction of transient changes in neurotransmitter concentration from dynamic PET data. IEEE Trans Med Imaging. 2007;26:359-373.
69. Wanat MJ, Willuhn I, Clark JJ, Phillips PEM. Phasic dopamine release in appetitive behaviors and drug addiction. Curr Drug Abuse Rev. 2009;2:195-213.

70. Heien MLAV, Khan AS, Ariansen JL, et al. Real-time measurement of dopamine fluctuations after cocaine in the brain of behaving rats. Proc Natl Acad Sci USA. 2005; 102:10023-10028.

71. Jeong J, Shi W-X, Hoffman R, et al. Bursting as a source of non-linear determinism in the firing patterns of nigral dopamine neurons. Eur J Neurosci. 2012;36:3214-3223.

72. Lewis LD, Setsompop K, Rosen BR, Polimeni JR. Fast fMRI can detect oscillatory neural activity in humans. Proc Natl Acad Sci USA. 2016;113:E6679-E6685.

73. Chen KT, Gong E, de Carvalho Macruz FB, et al. Ultra-low-dose ${ }^{18} \mathrm{~F}$-florbetaben amyloid PET imaging using deep learning with multi-contrast MRI inputs. Radiology. 2019;290:649-656.

74. Catana C. The dawn of a new era in low-dose PET imaging. Radiology. 2019;290: 657-658.

75. Catana C, Guimaraes AR, Rosen BR. PET and MR imaging: the odd couple or a match made in heaven? J Nucl Med. 2013;54:815-824.

76. Huppert TJ, Hoge RD, Diamond SG, Franceschini MA, Boas DA. A temporal comparison of BOLD, ASL, and NIRS hemodynamic responses to motor stimuli in adult humans. Neuroimage. 2006;29:368-382.

77. White BR, Snyder AZ, Cohen AL, et al. Resting-state functional connectivity in the human brain revealed with diffuse optical tomography. Neuroimage. 2009;47: $148-156$.

78. Belliveau JW, Kennedy D, McKinstry R, et al. Functional mapping of the human visual cortex by magnetic resonance imaging. Science. 1991;254:716-719. 